\title{
Pure Obsessive Compulsive Disorder in Three Generations
}

\author{
Alireza Rahimi ${ }^{1}$; Mohammad Haghighi ${ }^{1}$; Farshid Shamsaei ${ }^{1, *}$ \\ ${ }^{1}$ Research Center for Behavioral Disorders and Substance Abuse, Hamadan University of Medical Sciences, Hamadan, IR Iran \\ *Corresponding author: Farshid Shamsaei, Research Center for Behavioral Disorders and Substance Abuse, Hamadan University of Medical Sciences, Hamadan, IR \\ Iran. Tel: +98-8138232193, Fax: +98-8138380447, E-mail: shamsaei@umsha.ac.ir \\ Received: November 2, 2013; Revised: July 14, 2014; Accepted: August 22, 2014
}

\begin{abstract}
Introduction: Obsessive-compulsive disorder $(\mathrm{OCD})$ is a psychiatric disorder, which has been shown to affect 2 - 3.5\% of people, during their lifetimes. Identification of familial more homogenous characteristics of OCD may help to define relevant subtypes and increase the power of genetic and neurobiological studies of OCD.

Case Presentation: This case report describes an adult woman suffering from symptoms of energy loss, insomnia, lack of appetite, and depressed mood. The patient history was positive for counting coercion. The patient's genogram revealed counting coercion in three generations of her family.

Conclusions: This case highlights the issue whether counting can be a distinctive feature among inflicted and not inflicted individuals, such as hoarding. Also, it is still unclear what is it really transferred; the vulnerability to disease, which is transferred among three generations, or the symptoms of counting itself, by genes. Further studies are required to answer the debates on this issue.
\end{abstract}

Keywords: Antidepressive Agents; Obsessive-Compulsive Disorder; Mental Disorders

\section{Introduction}

Obsessive-compulsive disorder (OCD) is characterized by recurrent intrusive thoughts, images, or urges (obsessions) that typically cause anxiety or distress, and by repetitive mental or behavioral acts (compulsions) that the individual feels, driven to perform, either in response to an obsession, or according to rules that he or she believes must be applied rigidly. The OCD typically starts in childhood or adolescence, persists throughout a person's life, and produces substantial impairment in functioning due to the severe and chronic nature of the illness (1). Females are affected at a slightly higher rate than males in adulthood, although males are more commonly affected in childhood (1).

The OCD occurs worldwide, with common features across diverse ethnic groups and cultures. It affects approximately $2 \%$ of the population and is associated with substantial social, personal, and work impairment (2, 3). In fact, the World Health Organization (WHO) identified OCD among the top 20 causes of years of life lived with disability for 15 - 44-year-olds (4). Although generally longitudinally stable, OCD is known for its substantial heterogeneity; as symptom presentations and comorbidity patterns can vary markedly in different individuals (4).

The OCD is a clinically heterogeneous illness of unknown etiology, and there is considerable variation among patients in the types of obsession and compulsion, with which they present. The OCD is a neuropsy- chiatric condition characterized by recurrent, intrusive thoughts (obsessions) or repetitive behaviors that an individual feels driven to perform (compulsions). According to the WHO, OCD is among the 10 most disabling medical conditions (5).

In familial studies of OCD, the disorder is found to be five to seven fold more frequent in relatives of cases than in those of control subjects (5). Currently, there is a marked interest in grouping individuals with OCD, according to the categories of obsessions and compulsions they exhibit, or alternatively, in measuring scores on symptom factors or dimensions that vary between individuals with the disorder (6). Because OCD patients are rarely monosymptomatic, it has been difficult to recruit "pure" cases of OCD subtypes for genetic studies, and the possible familial aggregation of such potential clinical subtypes has not been demonstrated (7).

Identification of familial more homogenous characteristics of OCD may help to define relevant subtypes and increase the power of genetic and neurobiological studies of OCD. Although factor-analytic studies have found consistent clinically meaningful OCD symptom dimensions, there have been only limited attempts to evaluate the familiarity and potential genetic basis of such dimensions (8). We describe a case of counting compulsion in three generations of a family, pure case of OCD that may help in accumulating knowledge about the pathophysiology of the OCD.

Copyright ( ) 2015, Mazandaran University of Medical Sciences. This is an open-access article distributed under the terms of the Creative Commons Attribution-NonCommercial 4.0 International License (http://creativecommons.org/licenses/by-nc/4.0/) which permits copy and redistribute the material just in noncommercial usages, provided the original work is properly cited. 


\section{Case Presentation}

A 45-year-old married woman was followed as an outpatient at Farshchian Psychiatry Hospital in Hamadan, Iran in 2012. She referred to the clinic with symptoms of energy loss, insomnia, lack of appetite and depressed mood. The patient history was positive for counting coercion. She mentioned that she had these symptoms for many years, as she remembered, to various degrees. Counting coercion was found in the patient's sister biography. To survey these symptoms more accurately, we examined all the patient's family members, by requesting permission from the patient and taking her consensus; the results of which were recorded in the following genogram. As shown in the genogram, counting coercion is observed in three generations of this family. It is worth mentioning that none of the family members was treated for this disease. All mentioned that they had these symptoms since the early times; they did not have any other psychiatric disorder, based on our assessment. The other interesting point is that several of these persons performed counting in terms of even numbers and others, in terms of odd numbers (Figure 1).

\subsection{Family History}

The patient's mother had one serious suicide attempt at age 75 and was diagnosed with major depressive disorder with psychotic features and OCD. She also had noninsulin dependent diabetes mellitus. Also, her sister was treated for OCD as an out-patient for the past 8 years. Her father is alive and well.

\subsection{Mental Status Examination}

The patient was a thin, bleached woman, who appeared her stated age. She was dressed in skin tight, provocative clothing, costume jewelry earrings that eclipsed her ears and hung to her shoulders, heavy make-up and elaborately styled hair. She had difficulty sitting still and fidgeted constantly in her chair. Her speech was rapid, mildly pressured, and she rarely finished a sentence. She described her mood as "anxious".

Her effect appeared anxious. Her thought processes showed mild circumstantiality and tangentially. More significant was her inability to finish a thought, as exhibited by her incomplete sentences. Her thought content was focused upon social relationship themes. She denied suicidal or homicidal ideations, or any auditory or visual hallucination. Cognitive testing was not formally done.

\subsection{Past Psychiatric History}

Over the years, the patient had been maintained on various antidepressant and antianxiety agents. These included phenelzine, trazodone, desipramine, alprazolam, clonazapam, and hydroxyzine. Currently, she was on fluoxetine $20 \mathrm{mg}$ daily and clonazeparn $0.5 \mathrm{mg}$, twice a day, and $1.0 \mathrm{mg}$ at bedtime. The antidepressants had been effective over the years in treating her depression. She has never used more clonazepam than prescribed, and there was no history of substance abuse. Furthermore, there was no history of discreet manic episodes, and she was never treated with neuroleptics.

\section{Discussion}

The mechanisms by which OCD is transmitted from parent to child have not been identified, although they are likely to be both genetic and environmental, or due to the interaction between individual vulnerability factors (genetics) and environmental factors (e.g. acute and chronic stressors, maladaptive parenting). Identifying etiological influences on OCD is challenging, as OCD is a clinically and etiologically heterogeneous disorder (9).

A complex segregation analysis provided evidence supporting major gene effects and other familial effects in OCD vulnerability, with respect to familial aggregation of OCD (10).

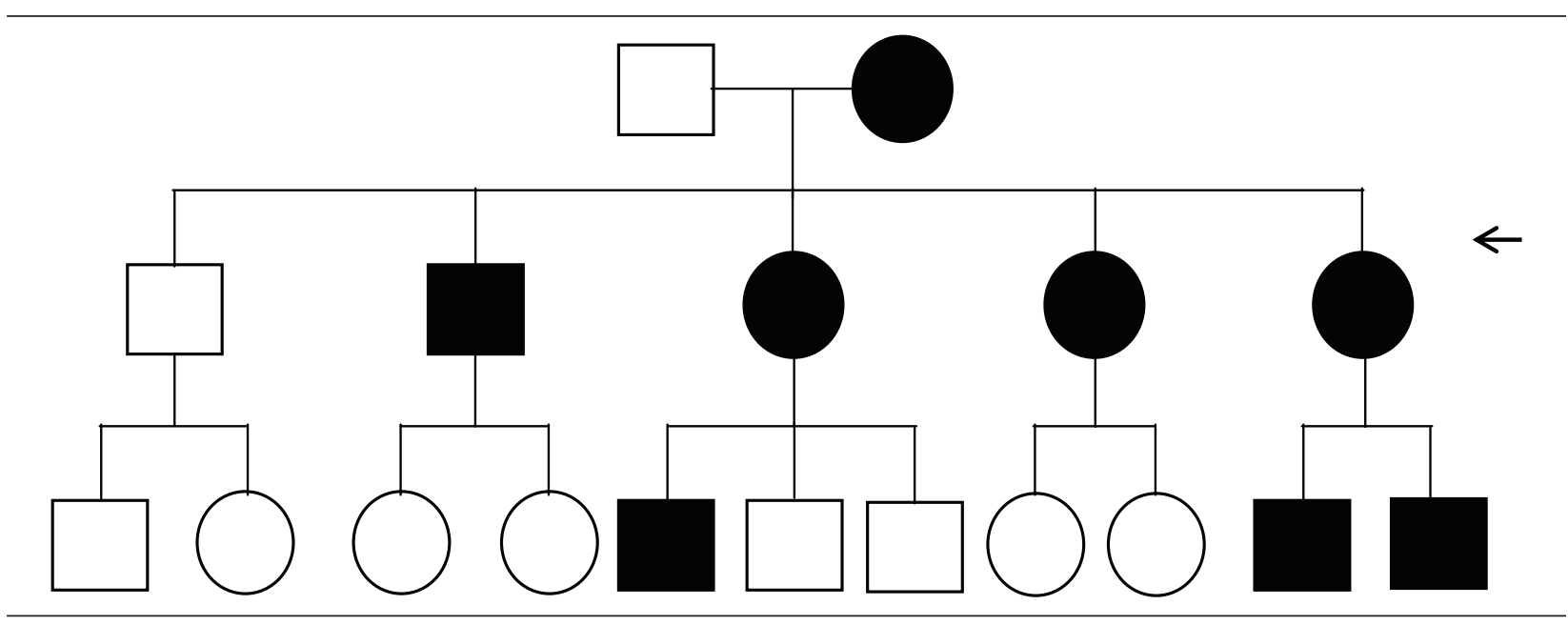

Figure 1. Family Genogram: Saliva Material Available From OCD (Obsessive-Compulsive Disorder)-Affected Sample: I - 2, II - 2, II - 3, II - 5 (index case), III - 5 
A significant amount of research attests to the role of heredity in OCD, and in addition, a range of hypothesized biological factors $(8,11)$, shared personality factors may account for the increased prevalence rates of OCD among first-degree relatives of OCD probands. Researchers have increasingly focused their attention on what is inherited. Phenotypic heterogeneity is increasingly recognized as a major impediment to the elucidation of the pathophysiology and etiology of neuropsychiatric disorders (12). Although standard classification systems, such as the Fifth Edition Diagnostic and Statistical Manual of Mental Disorders and International Classification of Diseases-10, regard OCD as a unitary nosological entity, there is increasing evidence that this severe and potentially disabling condition is phenotypically heterogeneous (6). However, comprising a new chapter on Obsessive-Compulsive and Related Disorders in the fifth edition of the Diagnostic and Statistical Manual of Mental Disorders (DSM-5) reflects the increasing evidence of OCD relatedness to other disorders such as body dysmorphic disorder, trichotillomania, hoarding disorder and excoriation disorder (13).

Using a family study design, with matched control subjects, and applying operational criteria for the assessment of OCD, McKeon and Murray (14) found no increased familial risk of $O C D$, even though they did observe a greater rate of mental illness in the first-degree relatives of OCD probands. Bellodi et al. (15), using no comparison group, found a low prevalence of OCD (3.4\%) among first-degree relatives. In cases with an early onset (before age 14 ), prevalence reached $8.8 \%$, which seems to indicate a biological vulnerability. Grabe et al. reported that Cox proportional hazard analyses revealed a 6.2-fold higher risk (hazard ratio) for relatives of all OCD cases, for definite OCD, and a 2.2-fold higher risk, for subclinical OCD, compared with relatives of comparison subjects (16). Clinical features may correlate with course, prognosis and treatment responsiveness and it has been hypothesized that different clinical subgroups may result from different etiologies and pathogeneses (17).

Regarding the reported case, this issue is whether counting can be a distinctive feature among inflicted and no inflicted individuals, such as hoarding. Another question is "what is really transferred?" Is it this vulnerability to disease, which is transferred among three generations, or the symptoms of counting itself are transferred by genes. This theory requires confirmation from genetic studies in this field.

\section{Acknowledgements}

Authors of the study thank colleagues from the Research Center for Behavioral Disorders and Substance Abuse, Hamadan University of Medical Sciences, Hamadan, Iran, for providing source data files and further supporting data, concerning the sampling methodology.

\section{Authors' Contributions}

Farshid Shamsaei participated in designing the evaluation and all authors participated in interpretation of clinical data, drafting and revising the manuscript. Alireza Rahimi and Mohammad Haghighi collected the clinical data. All authors read and approved the final manuscript.

\section{Declaration of interest}

None declared.

\section{References}

1. Ruscio AM, Stein DJ, Chiu WT, Kessler RC. The epidemiology of obsessive-compulsive disorder in the National Comorbidity Survey Replication. Mol Psychiatry. 2010;15(1):53-63.

2. Angst J, Gamma A, Endrass J, Goodwin R, Ajdacic V, Eich D, et al. Obsessive-compulsive severity spectrum in the community: prevalence, comorbidity, and course. Eur Arch Psychiatry Clin Neurosci. 2004;254(3):156-64.

3. Kessler RC, Berglund P, Demler O, Jin R, Merikangas KR, Walters EE. Lifetime prevalence and age-of-onset distributions of DSM-IV disorders in the National Comorbidity Survey Replication. Arch Gen Psychiatry. 2005;62(6):593-602.

4. Murphy DL, Timpano KR, Wheaton MG, Greenberg BD, Miguel EC. Obsessive-compulsive disorder and its related disorders: a reappraisal of obsessive-compulsive spectrum concepts. Dialogues Clin Neurosci. 2010;12(2):131-48.

5. Hanna GL, Himle JA, Curtis GC, Gillespie BW. A family study of obsessive-compulsive disorder with pediatric probands. Am J Med Genet B Neuropsychiatr Genet. 2005;134B(1):13-9.

6. Mataix-Cols D, Rosario-Campos MC, Leckman JF. A multidimensional model of obsessive-compulsive disorder. Am J Psychiatry. 2005;162(2):228-38.

7. Emilien G, Dinan T, Lepola UM, Durlach C. Anxiety disorders: Pathophysiology and pharmacological treatment.Philadelphia: Springer; 2002.

8. Hasler G, Pinto A, Greenberg BD, Samuels J, Fyer AJ, Pauls D, et al. Familiality of factor analysis-derived YBOCS dimensions in OCDaffected sibling pairs from the OCD Collaborative Genetics Study. Biol Psychiatry. 2007;61(5):617-25.

9. Huntsman L. Parents with mental health issues: Consequences for children and effectiveness of interventions designed to assist children and their families.. Available from: http://www.community.nsw. gov.au/docswr/_assets/main/documents/research_parentalmentalhealth.pdf.

10. Nestadt G, Lan T, Samuels J, Riddle M, Bienvenu O3, Liang KY, et al. Complex segregation analysis provides compelling evidence for a major gene underlying obsessive-compulsive disorder and for heterogeneity by sex. Am J Hum Genet. 2000;67(6):1611-6.

11. Rector NA, Cassin SE, Richter MA, Burroughs E. Obsessive beliefs in first-degree relatives of patients with OCD: a test of the cognitive vulnerability model. J Anxiety Disord. 2009;23(1):145-9.

12. Baer L. Factor analysis of symptom subtypes of obsessive compulsive disorder and their relation to personality and tic disorders. JClin Psychiatry. 1994;55 Suppl:18-23.

13. American Psychiatric Association. Diagnostic and Statistical Manual of Mental Disorders (DSM-5).Washington DC: American Psychiatric Association; 2013.

14. McKeon P, Murray R. Familial aspects of obsessive-compulsive neurosis. BrJ Psychiatry. 1987;151:528-34.

15. Bellodi L, Sciuto G, Diaferia G, Ronchi P, Smeraldi E. Psychiatric disorders in the families of patients with obsessive-compulsive disorder. Psychiatry Res. 1992;42(2):111-20.

16. Grabe HJ, Ruhrmann S, Ettelt S, Buhtz F, Hochrein A, SchulzeRauschenbach $\mathrm{S}$, et al. Familiality of obsessive-compulsive disorder in nonclinical and clinical subjects. Am J Psychiatry. 2006;163(11):1986-92.

17. Miguel EC, Leckman JF, Rauch S, do Rosario-Campos MC, Hounie AG, Mercadante MT, et al. Obsessive-compulsive disorder phenotypes: implications for genetic studies. Mol Psychiatry. 2005;10(3):258-75. 